IdeAs

Idées d'Amériques

8 | 2016

Ressources minières dans les Amériques: Mutations

d'un continent

\title{
Ressources minières et énergétiques : le choc des discours
}

Sandrine Tolazzi

\section{OpenEdition}

Journals

Édition électronique

URL : https://journals.openedition.org/ideas/1685

DOI : $10.4000 /$ ideas. 1685

ISSN : 1950-5701

Éditeur

Institut des Amériques

Référence électronique

Sandrine Tolazzi, "Ressources minières et énergétiques : le choc des discours », IdeAs [En ligne], 8 |

2016, mis en ligne le 16 décembre 2016, consulté le 18 octobre 2022. URL : http://

journals.openedition.org/ideas/1685; DOI : https://doi.org/10.4000/ideas.1685

Ce document a été généré automatiquement le 18 octobre 2022.

\section{(c)}

Creative Commons - Attribution - Pas d'Utilisation Commerciale - Pas de Modification 4.0 International - CC BY-NC-ND 4.0

https://creativecommons.org/licenses/by-nc-nd/4.0/ 


\title{
Ressources minières et énergétiques : le choc des discours
}

\author{
Sandrine Tolazzi
}

Les deux ouvrages et la table ronde présentés ici permettront d'apprécier les divergences de points de vue et la pluralité des discours au sujet des ressources minières et énergétiques en fonction des disciplines, des orientations politiques, des intérêts et de la nature de l'objet ou du sujet impacté par le développement de ces ressources (environnement, communautés locales, économie, sécurité, etc.). Le premier, Vive les énergies fossiles! La contre-révolution énergétique (Bruxelles: Texquis, 2014) pourra paraître surprenant dans un contexte où la nécessité d'amorcer une transition énergétique semble faire consensus parmi les pays ayant conclu l'accord international sur le climat lors de la COP 21. Néanmoins, son auteur, Samuele Furfari, expert européen auprès de la Direction Générale de l'Énergie de la Commission européenne et professeur de géopolitique de l'énergie à l'Université Libre de Bruxelles, défend l'idée selon laquelle les énergies fossiles ont encore de beaux jours devant elles. Il est rejoint dans ses propos par les experts réunis au sein du Research Triangle Institute, institut de recherche indépendant chargé de conseiller des entreprises privées mais aussi le gouvernement américain lors de l'élaboration de politiques publiques, à l'occasion d'une table ronde sur l'impact du gaz et du pétrole de schiste (Washington, D.C, 22 septembre 2015). Ces experts semblent en effet mettre en avant les avantages du développement actuel du gaz et du pétrole de schiste aux États-Unis lors de leurs discussions sur les conséquences économiques, environnementales et géopolitiques de ce développement. Pourtant, le survol des enjeux relatifs au développement des ressources minières et énergétiques effectué par Norman Mousseau, professeur de physique et titulaire de la Chaire Université de Montréal «Matériaux complexes, énergies et ressources naturelles » dans son ouvrage Le défi des ressources minières (Québec, Multimondes, 2012), montre que les modèles d'exploitation actuels de ces ressources ne permettent pas de résoudre les problèmes posés, en particulier en ce qui concerne le développement durable. 
FURFARI, Samuele. Vive les énergies fossiles! La contre-révolution énergétique (Bruxelles : Texquis, 2014)

Dans cet ouvrage, Samuele Furfari replace le développement actuel des énergies fossiles (pétrole et gaz) dans une perspective historique qui permet de comprendre les espoirs nourris par ce développement au regard de la crise du pétrole amorcée dans les années 1970. Pour Furfari, nous arrivons au terme de cette pénible « quarantaine » (1973-2013) pendant laquelle les compagnies pétrolières se sont comportées en prédateurs par rapport aux pays producteurs (21) avant que ces derniers ne décident d'augmenter les prix et de diminuer leurs exportations, provoquant deux chocs pétroliers qui ont poussé les pays occidentaux à s'organiser (virage vers le nucléaire, nouvelles explorations). La période de calme du milieu des années 1980 a été suivie par une nouvelle période de volatilité des prix dans les années 1990 (crise asiatique), puis une flambée à partir de 2004 qui a fait craindre la « fin du pétrole».

Pour Furfari, ce spectre de la «fin du pétrole» s'éloigne aujourd'hui incontestablement, et ce en raison de quatre facteurs principaux :

- L'entrée en vigueur de la Convention sur le droit de la mer en 1994, qui a permis l'augmentation de la superficie des pays côtiers et le début de l'exploration dans les espaces maritimes (57-75).

- La découverte de nouveaux gisements pétrolifères et leur récente mise en production grâce aux investissements réalisés depuis 2004 (Canada, Mexique, Brésil) (77-136).

- La production de gaz conventionnel (Russie, États côtiers de la méditerranée occidentale) (137-176)

- La révolution du gaz de roche-mère (également appelé gaz de schiste) aux États-Unis (193-209), et la prospection ailleurs dans le monde. Furfari indique à ce sujet que, pour l'Union européenne, il est « de la plus haute importance stratégique de commencer à s'y intéresser très sérieusement » (227)

$5 \quad$ Les perspectives ouvertes par ces nouveaux développements permettent de résoudre les problèmes posés par une consommation mondiale d'énergie en hausse malgré les progrès réalisés dans le domaine de l'efficacité énergétique. Par ailleurs, les retombées économiques sont très importantes : pour les États-Unis, par exemple, l'abondance des énergies fossiles influe également sur de nombreux autres secteurs (industrie automobile, pâte à papier, engrais, etc.) et, selon Furfari, « la réduction des coûts de l'énergie va contribuer à revitaliser l'ensemble du secteur manufacturier aux États-Unis et aura un impact positif sur la position concurrentielle de l'industrie américaine dans son ensemble. » (297) Dans le domaine des transports, l'utilisation du gaz de rochemère peut freiner la trop grande dépendance aux produits pétroliers (313). Enfin, du point de vue géopolitique, Furfari entrevoit un rôle grandissant des États-Unis mais aussi du Canada et de l'Australie, qui possèdent d'immenses réserves énergétiques, et donc une "réoccidentalisation» du monde de l'énergie (349), ce qui l'amène à conclure : «en résumé, les grands vainqueurs sont les U.S.A. qui ont osé innover. Rien de nouveau sous le soleil » (352).

Certes, Furfari n'occulte pas entièrement les problèmes soulevés par l'exploitation des énergies fossiles. Il reconnaît ainsi que «l'exploitation du pétrole n'apporte pas nécessairement le bien-être matériel à toute la population, les femmes et les enfants continuent d'être exploités et les guerres civiles guettent, sans oublier les dégâts environnementaux» (91), mais il ne commente pas outre mesure. En revanche, il présente le gaz naturel comme une solution pour réduire les émissions de gaz à effet de 
serre. En réponse au fait que le gaz de roche-mère est devenu si peu coûteux aux ÉtatsUnis que lorsqu'il est produit en même temps que le pétrole de roche-mère, il est brûlé en torchères - ce qui génère tout de même 360 millions de tonnes de $\mathrm{CO} 2$, soit l'équivalent de 70 millions de voitures en circulation - l'auteur répond que si ce gaz n'était pas brûlé en torchères, il serait de toute façon brûlé ailleurs et produirait donc la même quantité de CO2 (97). Par ailleurs, les importantes quantités d'eau utilisées par la fracturation hydraulique - ce qui peut poser problème par exemple en Algérie seront compensées par la découverte de nouvelles réserves d'eau (220). En somme, l'auteur balaie tous les arguments évoqués par les opposants au développement du gaz de roche-mère. Ainsi, au sujet de la pollution des nappes phréatiques, "s'il y avait un danger systématique, ça se saurait depuis longtemps» (241) ou, par rapport au traitement des eaux usées produites par l'extraction, «ce traitement va requérir de l'énergie et [...] les produits extraits doivent être gérés comme des déchets industriels, mais cela génère de l'activité économique. C'est de la "green economy" sans subvention étatique!» (246). L'auteur se positionne clairement par rapport à l'impact du développement des énergies fossiles : "L'utilisation de l'énergie fossile, en dépit de son impact environnemental, a eu des répercussions extraordinairement positives sur nos conditions de travail, de vie et de santé. » (361)

7 Pourquoi de nombreux pays s'engagent-ils alors dans la voie de la transition énergétique? Pour Furfari, ce choix est avant tout idéologique. Ainsi, « les idéologies à la mode aujourd'hui sont l'interventionnisme humanitaire et l'environnementalisme, toutes deux portées par des élans apparents de générosité d'ONG politisées » (11). La protection de l'environnement relèverait donc d'une idéologie qui, après la chute du mur de Berlin, est venue remplacer l'idéologie communiste, et s'est développée tout au long des années 1990 en influençant progressivement les instances dirigeantes (40). L'Agenda 21 adopté lors du Sommet de la Terre de 1992 est ainsi décrit comme une "dictature verte", tandis que le recours aux biocarburants a produit des résultats inverses de ceux escomptés, ce qui montre selon l'auteur que «les choix politiques en matière de politique énergétique peuvent s'avérer complètement erronés » (46), et l'amène à conclure que «manipuler le vaste marché de l'énergie est extrêmement complexe, aléatoire et pour tout dire souvent très improductif» (46). Le lecteur comprend aisément que Furfari part d'un point de vue néo-libéral lorsqu'il dénonce « la partie agissante de la population de nombreux pays » qui est «parvenue à prendre le pouvoir médiatique et éducatif et, ainsi, à museler l'esprit d'initiative qui pourrait perturber la sérénité qui lui est si précieuse » (353). Il ira jusqu'à justifier le fait qu'il parle très peu de changement climatique en arguant qu'il se « refuse à apporter de l'eau au moulin du mythe de l'origine anthropique du changement climatique » (356).

8 L'ouvrage de Samuele Furfari est ainsi emblématique de la manière dont l'appartenance à un courant politique et idéologique peut venir en appui d'un positionnement par rapport à la question du développement des ressources énergétiques, ce qui n'est pas sans intérêt puisque cet auteur est notamment expert auprès de la Commission européenne sur les aspects énergétiques.

RTI International. «Shale Oil and Gas : Impact on the Economy, Environment, and National Security ». The National Press Club, Washington, D.C., September 22, 2015. https://www.youtube.com/watch?v=-ZzuoB6rKo8 (consulté le 12 juillet 2016). 
10 L'institut de recherche indépendant Research Triangle International fournit des services de recherche aux gouvernements et aux entreprises de plus de 40 pays dans divers domaines (santé, politique, technologie, éducation, etc.). La table ronde organisée par cet institut le 22 septembre 2015 à Washington, D.C. a réuni plusieurs experts autour de la question de l'impact du gaz et pétrole de schiste sur l'économie, la sécurité et l'environnement aux États-Unis. Dans un contexte de développement croissant de cette ressource (plus de $71 \%$ d'augmentation de la production en quatre ans) qui a permis de réduire les importations de pétrole et de faire baisser les prix des énergies fossiles, le modérateur de cette table ronde, Vikhram Rao (auteur de Shale Gas: The Promise and the Peril. Research Triangle Park, N.C.: Research Triangle Institute Press, 2012), souhaite mettre en regard les atouts liés à ce développement en termes d'économie et de sécurité et les risques en termes de santé publique et d'environnement. Ainsi, l'augmentation de la production de gaz de schiste a eu pour effet de réduire la concurrence des pays d'Europe et d'Asie dans de nombreux secteurs (le gaz étant utilisé, par exemple, pour fabriquer des produits agricoles), de réduire les taux d'émission de $\mathrm{CO} 2$, et de renforcer le dollar. Mais les enjeux environnementaux sont également importants : chaque puits utilise jusqu'à 30 millions de litres d'eau, qui doit ensuite être recyclée en raison des produits toxiques qu'elle contient, le méthane produit se disperse dans l'environnement, et les tremblements de terre liés au processus de fracturation hydraulique sont de plus en plus fréquents.

11 Le premier intervenant, Greg Dolan, est le président-directeur général du Methanol Institute, une association qui regroupe des entreprises productrices de méthanol, un produit dérivé du gaz naturel et utilisé comme solvant dans la fabrication de nombreux produits. Selon lui, la révolution du gaz de schiste a conduit à une baisse significative des prix et à l'augmentation de la production de méthanol. De nombreuses installations ont rouvert, tandis que d'autres voyaient le jour, créant des milliers d'emplois et de recettes fiscales. Parallèlement à cela, de nouvelles usines de produits chimiques utilisant le méthanol sont en construction, ce qui représente 284000 emplois additionnels aux États-Unis. Enfin, le méthanol peut être mélangé à de l'essence et être utilisé dans le secteur des transports. Le Methanol Institute a ainsi déposé un projet de loi visant à encourager les constructeurs automobiles à produire des voitures pouvant accepter plusieurs types de carburant (essence ou essence mélangée au méthanol). Ce sont donc les avantages économiques du développement du gaz de schiste qui sont soulignés ici, ce qui n'est guère surprenant de la part d'un expert qui représente avant tout un groupe de pression financé par les industries productrices de méthanol.

12 L'intervenant suivant, Brian Southwell, est spécialiste de la communication et des comportements humains. Enseignant-chercheur à l'Université Duke et l'Université de Caroline du Nord à Chapel Hill, il dirige également le programme « Science in the Public Sphere " (la science dans la sphère publique au sein du groupe Research Triangle International. Son intervention concerne l'opinion publique par rapport à la question du gaz de schiste, qui selon lui dépend beaucoup de l'information qui circule. Les travaux menés dans ce domaine indiquent notamment une méconnaissance des processus d'exploration et d'extraction, mais également un soutien en faveur d'une réglementation stricte de l'industrie. Southwell pointe aussi du doigt les faiblesses de certaines études. Par exemple, beaucoup de travaux se sont concentrés sur des zones bien spécifiques (zones concernées par l'extraction), ce qui ne reflète pas l'opinion américaine dans son ensemble. L'utilisation de questionnaires ne permet pas d'obtenir 
la même qualité de réponse que des entretiens dirigés ou semi dirigés. Certains termes, en particulier celui de "fracturation hydraulique », sont chargés d'un sens négatif, et peuvent donc produire des réponses différentes par rapport à des termes plus «neutres ». Pour conclure, Southwell insiste sur la nécessité de mieux étudier le lien entre les informations perçues et reçues par le public et les attitudes de ce dernier visà-vis de la question du développement du gaz de schiste. Il part du principe que plus les informations auxquelles sont exposés les individus seront positives, plus ces derniers auront des attitudes positives, ce qui envoie un message clair aux groupes de pression liés à l'industrie en soulignant l'importance de leur travail, notamment auprès des médias.

13 La troisième intervenante, Anne Korin, est co-directrice de l'Institute for the Analysis of Global Security, un groupe de réflexion sur l'énergie et la sécurité, et conseillère auprès du United States Energy Security Council, dont l'objectif est de réduire la dépendance des États-Unis au pétrole. Sa contribution concerne essentiellement les aspects stratégiques et géopolitiques du développement du gaz de schiste aux ÉtatsUnis comme alternative au pétrole dans le secteur des transports. En effet, à l'heure actuelle, ce sont toujours les pays de l'OPEP qui fixent les prix du pétrole en agissant sur les volumes qu'ils décident de vendre. Ils choisissent de maintenir les prix bas pour deux raisons principales. La première est la volonté d'affaiblir l'industrie américaine, puisqu'il en coûte plus aux États-Unis que dans ces pays de produire du pétrole, et que l'opération n'est plus rentable en dessous d'un certain prix. La seconde est que l'Arabie Saoudite maintient ainsi une pression sur l'Iran, qui aurait également besoin d'une hausse des prix pour équilibrer son budget, mais qui menace de s'engager dans le nucléaire. L'ombre d'une guerre nucléaire entre sunnites et chiites semble donc planer dans la région, ce qui ne présage rien de bon pour le marché du pétrole. Dans ce contexte, le recours au gaz de schiste comme alternative au pétrole est très important pour la sécurité de ce qu'Anne Korin appelle "le monde libre», et permettra de maintenir le prix du pétrole à un niveau bas puisque le gaz entrera en compétition avec le pétrole dans le domaine des transports.

On remarque ici très clairement que les positions des experts de ce panel sur le gaz de schiste sont étroitement liées à leur statut et à leur orientation politique d'une part, et qu'ils ont probablement été choisis par rapport à un public que l'on suppose principalement composé de personnes appartenant à l'industrie du gaz de schiste (c'est le cas de celles qui sont intervenues lors de la période des questions). Contrairement au modérateur du panel, on ne sera pas vraiment surpris du fait qu'aucune question n'a véritablement concerné l'impact environnemental de cette industrie, impact qui n'a pas non plus été réellement discuté par les experts. À ce sujet, sa brève conclusion consiste à affirmer que l'élaboration et le respect d'une réglementation adaptée devraient suffire à réduire cet impact.

MOUSSEAU, Normand. Le défi des ressources minières (Québec : Multimondes, 2012).

L'impact économique, géostratégique et environnemental du développement des ressources minières et énergétiques est également abordé au travers de l'ouvrage de Normand Mousseau, physicien théoricien canadien qui a notamment co-présidé la Commission sur les enjeux énergétiques du Québec dont le rapport a été publié en 2014. Mousseau souhaite aborder les principaux enjeux qui sous-tendent l'exploitation des ressources minières depuis le regain de l'industrie extractive lié à l'explosion du prix des métaux et du pétrole entre 2002 et 2008 (1). 
17 L'auteur revient d'abord sur des aspects géologiques et métallurgiques - naissance de la métallurgie, puis avènement de l'Âge de l'acier avec la révolution industrielle et utilisation d'un nombre croissant d'éléments naturels - pour expliquer l'augmentation importante de la demande et les tensions que cela peut générer. En effet, bien que l'industrie améliore constamment son bilan énergétique au niveau de l'extraction et de la transformation, ce bilan est contrecarré par l'augmentation de la production et l'appauvrissement des gisements (ce qui nécessite davantage d'énergie pour l'extraction) (32-33). Dans ce contexte, le coût environnemental de la production est également de plus en plus important. Le défi, alors que la quantité des ressources disponibles est difficile à évaluer, consiste donc à développer des usages mieux contrôlés du point de vue environnemental et à mettre en place des solutions de remplacement pour les éléments les plus rares (56).

18 Mousseau aborde ensuite les enjeux économiques liés à l'industrie minière dont le modèle néolibéral est aujourd'hui largement critiqué. Les économistes marxistes y voient en effet la manifestation de l'exploitation des travailleurs locaux et des ressources non renouvelables par de gros investisseurs étrangers, ce qui justifie à leurs yeux la nationalisation des ressources (82). Pour les scientifiques et environnementalistes, c'est l'absence de prise en compte des concepts de développement durable et social et de la nature cyclique de l'industrie qui pose aujourd'hui problème (81). Sous la pression des critiques, les grandes institutions telles que la Banque mondiale, suivies par l'industrie elle-même, proposent un modèle libéral "doux» qui favorise le développement équitable et diminue la pauvreté (83), et adoptent un discours rassurant sur le développement durable, ce dernier étant limité à la mise en place d'une économie diversifiée survivant à la fermeture des mines. Mais cela ne diminue aucunement la fusion/acquisition qui continue de concentrer le secteur autour de quelques multinationales déconnectées des mines et des travailleurs (96). Les ajustements du modèle néolibéral se font à la marge, et ne répondent pas aux questions de propriété, de contrôle de la production, de responsabilité par rapport aux conséquences environnementales, de calculs de redevances, de corruption, et de coûts sociaux. L'auteur conclut que « la mise en application des concepts de développement durable pour l'exploitation des ressources naturelles non renouvelables est un défi qui n'a pas encore de solution » (110).

19 Ce propos est illustré à travers plusieurs exemples de gestion des ressources naturelles. En Australie, le développement du secteur minier a fait augmenter le dollar australien et diminuer les secteurs manufacturier et touristique en conséquence. Les revenus miniers sont directement incorporés au budget de l'État plutôt que d'être mis de côté, pendant que la base industrielle du pays s'effrite, ce qui laisse entrevoir une crise de grande ampleur lors du prochain effondrement minier (143). Le Canada, qui est devenu un paradis minier fiscal et légal, met également peu d'argent de côté pour diversifier son économie. Pourtant, certains pays comme l'Indonésie ont réussi à mettre en place une législation qui oblige les entreprises à céder une partie de leurs actifs à des intérêts indonésiens, à transformer une certaine proportion du minerai brut sur place, ou à embaucher des travailleurs locaux dans le cadre d'un permis d'exploration qui est limité à la fois géographiquement et dans la durée (143-144).

Mousseau évoque enfin dans la dernière partie de son ouvrage les grands défis pour le secteur minier. Le premier est lié au caractère cyclique du secteur, qui réagit actuellement à la transformation de l'économie mondiale en raison d'une forte 
demande des pays émergents comme la Chine, mais sera en perte de vitesse s'il y a fléchissement de cette demande. Dès lors, il semble dangereux pour les pays souhaitant reconstruire leur économie en s'appuyant sur l'industrie minière de s'engager dans cette voie. Ensuite, l'exploitation de gisements à teneur toujours plus faible a un coût environnemental et social croissant que la société a de plus en plus de mal à accepter, ce qui nécessite l'émergence d'un nouveau paradigme intégrant notamment les ressources renouvelables et le recyclage des matériaux. Enfin, la mise en œuvre d'un nouveau modèle minier avec une présence forte du gouvernement qui permette d'investir dans des fonds souverains pour éviter la tentation des dépenses immédiates et de protéger efficacement les communautés locales et l'environnement est nécessaire avec, à terme, l'élaboration d'une stratégie mondiale des ressources minières.

21 L'analyse de ces trois documents montre que la multiplicité des discours et des points de vue au sujet de l'exploitation des ressources minières est fortement liée à des prises de position politiques et idéologiques d'une part, et au statut de celui qui produit le discours (représentant de l'industrie, chercheur au service de l'industrie ou rattaché à l'université, conseiller du gouvernement, etc.) d'autre part. On remarque également, de manière plus générale, qu'il paraît impossible de réconcilier la vision à court terme de l'industrie et la notion de développement durable - ce que souligne Mousseau dans son ouvrage. L'intervention étatique semble être un élément clé pour infléchir la donne, mais si l'on prend l'exemple des États-Unis, les atouts économiques et géostratégiques dans un contexte de crise économique et de menace sécuritaire croissante s'opposent à un contrôle plus strict de l'industrie qui pourrait freiner son développement. Ainsi, il n'est guère surprenant que le discours environnementaliste des uns se heurte au réalisme libéral des autres.

\section{AUTEUR}

\section{SANDRINE TOLAZZI}

Sandrine Tolazzi est maître de conférences à l'Université Grenoble Alpes (UFR des Langues, ILCEA4 - Institut des Langues et des Cultures d'Europe, Amérique, Afrique, Asie et Australie). sandrine.tolazzi@univ-grenoble-alpes.fr 